\title{
Flemish Language
}

National Cancer Institute

\section{Source}

National Cancer Institute. Flemish Language. NCI Thesaurus. Code C154055.

A West Germanic language native to Netherlands and Flanders. 